\title{
Review of: "Disorganization of intercalated discs in dilated cardiomyopathy"
}

\author{
Amr Zaher ${ }^{1}$ \\ 1 Zewail City of Science and Technology
}

Potential competing interests: The author(s) declared that no potential competing interests exist.

\begin{abstract}
Dilated cardiomyopathy (DCM) is a primary myocardial disease, the pathology of which is left ventricular or biventricular dilation and impaired myocardial contractility. The clinical and pathological diagnosis of DCM is difficult, and other cardiac diseases must be ruled out. Several studies have reported pathological findings that are characteristic of DCM, including cardiomyocyte atrophy, nuclear pleomorphism, and interstitial fibrosis, but none of these findings are DCM-specific. In this study, we examined the morphological differences in the intercalated discs (ICDs) between three groups of patients, a DCM group, a chronic heart failure group, and a control group. A total of 22 autopsy cases, including five DCM cases, nine CHF cases and eight control cases, were retrieved from the archives of the Department of Pathology at Akita University, Japan. The morphological differences were examined using multiple methods: macroscopic examination, light microscopy, immunohistochemistry, electron microscopy, and gene expression analyses. We observed disorganized ICDs, clearly illustrated by $\mathrm{N}$-cadherin immunostaining in the DCM group. "Reduction of N-cadherin immunostaining intensity" and "ICD scattering" was DCMspecific. The results suggest that disorganized ICDs contribute to the development of DCM, and that Ncadherin immunostaining is useful for determining the presence of disorganized ICDs and for the pathological diagnosis of DCM.
\end{abstract}

\section{Review:}

The results of this research are interesting and can contribute to the development of DCM-specific diagnosis assays. Further investigation into the molecular basis of DCM development such as the involvement of more extracellular proteins including integrins and laminin as well as the sarcomeric structures of cardiomyocytes would give a deeper insight into the DCM development and hence better DCM diagnosis. 